\title{
Designing Types for R, Empirically
}

\author{
ALEXI TURCOTTE, Northeastern University, USA \\ AVIRAL GOEL, Northeastern University, USA \\ FILIP KŘIKAVA, Czech Technical University, Czechia \\ JAN VITEK, Northeastern University, USA and Czech Technical University, Czechia
}

\begin{abstract}
The R programming language is widely used in a variety of domains. It was designed to favor an interactive style of programming with minimal syntactic and conceptual overhead. This design is well suited to data analysis, but a bad fit for tools such as compilers or program analyzers. In particular, $\mathrm{R}$ has no type annotations, and all operations are dynamically checked at runtime. The starting point for our work are the two questions: what expressive power is needed to accurately type $R$ code? and which type system is the $R$ community willing to adopt? Both questions are difficult to answer without actually experimenting with a type system. The goal of this paper is to provide data that can feed into that design process. To this end, we perform a large corpus analysis to gain insights in the degree of polymorphism exhibited by idiomatic R code and explore potential benefits that the $\mathrm{R}$ community could accrue from a simple type system. As a starting point, we infer type signatures for 25,215 functions from 412 packages among the most widely used open source $\mathrm{R}$ libraries. We then conduct an evaluation on 8,694 clients of these packages, as well as on end-user code from the Kaggle data science competition website.
\end{abstract}

CCS Concepts: • Software and its engineering $\rightarrow$ Language features; General programming languages.

Additional Key Words and Phrases: type declarations, dynamic languages, $\mathrm{R}$

\section{ACM Reference Format:}

Alexi Turcotte, Aviral Goel, Filip Křikava, and Jan Vitek. 2020. Designing Types for R, Empirically. Proc. ACM Program. Lang. 4, OOPSLA, Article 181 (November 2020), 25 pages. https://doi.org/10.1145/3428249

\section{INTRODUCTION}

Our community builds, improves, and reasons about programming languages. To make design decisions that benefit users, we need to understand our target language as well as the real-world needs it answers. Often, we can appeal to intuition, as many languages are intended for general purpose programming tasks. Unfortunately, intuition may fail us when looking at domain-specific languages designed for a particular group of users to solve specific needs. This is the case of the data science language R.

$\mathrm{R}$ and its ancestor $\mathrm{S}$ were designed, implemented, and maintained by statisticians. Originally they aimed to be glue languages for statistical routines written in Fortran. Over three decades they became widely used across many fields for exploratory data analysis. Modern $\mathrm{R}$ is fascinating as an object of study. It is a vectorized, dynamically typed, lazy functional language with limited side-effects, extensive reflective facilities and retrofitted object-oriented programming support.

Many of the design decisions that gave us $\mathrm{R}$ were intended to foster an interactive and exploratory programming style. These include, to name a few salient ones, the lack of type annotations, the

Authors' addresses: Alexi Turcotte, Northeastern University, USA; Aviral Goel, Northeastern University, USA; Filip Křikava, Czech Technical University, Czechia; Jan Vitek, Northeastern University, USA, Czech Technical University, Czechia.

This work is licensed under a Creative Commons Attribution 4.0 International License.

(C) 2020 Copyright held by the owner/author(s).

2475-1421/2020/11-ART181

https://doi.org/10.1145/3428249

Proc. ACM Program. Lang., Vol. 4, No. OOPSLA, Article 181. Publication date: November 2020. 
ability to use syntactic shortcuts, and widespread conversion between data types. While these choices have decreased the barrier to entry-many data science educational programs do not teach $\mathrm{R}$ itself but simply introduce some of its key libraries-they also allow for errors to go undetected.

Retrofitting a type system to the R language would increase our assurance in the result of data analysis, but this requires facing two challenges. First, it is unclear what would be the right type system for a language as baroque as R. For example, one of the most popular data type, the data. frame, is manipulated through reflective operations-a data frame is a table whose columns can be added or removed on the fly. Second, but just as crucially, designing a type system that will be adopted would require overcoming some prejudices and educating large numbers of users.

The goal of this paper is to gather data that can be used as input to the process of designing a type system for R. To focus our work, we chose to limit the scope of investigation on the design of a language for function type signatures. Thus, this paper will use the collected data to document the signatures of user-defined functions. For this, we design a simple type language that matches the $\mathrm{R}$ data types. We then extract call and return types from execution traces of a corpus of widely used libraries, and finally synthesize type signatures. This allows us to see how far one can get with a simple type language and identify limitations of our design. We validate the robustness of the extracted type signatures by implementing a contract checker that weaves types around their respective functions, and use end-user code and clients of the target packages for validation.

To sum up, we make the following contributions:

- We implemented scalable and robust tooling to automatically extract type signatures and instrument $\mathrm{R}$ functions with checks based on their declared types.

- We carried out a corpus analysis of 412 widely used and maintained packages to synthesize function type signatures and validated the robustness of the signatures against 160,379 programs that use those functions.

- We report on the appropriateness and usefulness of a simple type language for $\mathrm{R}$.

- Our data and code are open source and publicly available. ${ }^{1}$

\section{BACKGROUND}

This section introduces related work and gives a short primer on R.

\subsection{Related Work}

Dynamic programming languages such as Racket, JavaScript, PHP and Lua have been extended post factum with static type systems. In each case, the type system was carefully engineered to match the salient characteristics of its host language and to foster a particular programming style. For example, Typed Racket emphasizes functional programming and migration from untyped to fully typed code [Tobin-Hochstadt and Felleisen 2008], Hack [Verlaguet 2013] and TypeScript [Bierman et al. 2014] focus on object-oriented features of PHP and JavaScript, respectively. They allow users to mix typed and untyped code in a fine-grained manner. In the case of Lua [Maidl et al. 2014], the type system tried to account for the myriad ways Lua programmers use tables. Other languages adopted a mix of typed and untyped code by design. In Julia, type annotations are needed for method dispatch and performance [Bezanson et al. 2018]. In Thorn, users could freely move between typed and untyped code thanks to the addition of like types [Wrigstad et al. 2010]. Lastly C\# is an example of a statically typed language which added a dynamic type [Bierman et al. 2010].

But what if the design of the type system is unclear? Andreasen et al. [2016] propose a promising approach called trace typing. With trace typing, a new type system can be prototyped and evaluated

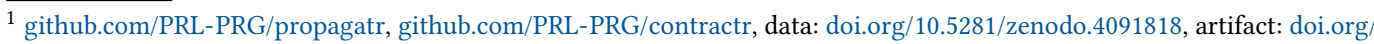
10.5281/zenodo.4037278
} 
by applying the type rule to execution traces of programs. While the approach has the limitation of dynamic analysis techniques, namely that the results are only as good at the coverage of the source code, it allows one to quickly test new design and quantify how much of a code base can be type-checked. Other approaches that infer types for dynamic analysis include the work of Furr et al. [2009] and An et al. [2011] for Ruby.

Recent work has gone into adding types to Python, the other eminent data science language. Typilus is an interesting piece of recent work which explores using neural networks to infer types for Python programs [Allamanis et al. 2020], and Python itself added support for type hints in Python 3.5 [Python Team 2020]. There is no previous work on types for R. We take inspiration in the aforementioned works but focus on adapting them to our target language.

\subsection{The R Language}

The R Project is a key tool for data analysis. At the heart of $\mathrm{R}$ is a vectorized, dynamic, lazy, functional, object-oriented language with an unusual combination of features [Morandat et al. 2012]. R was designed by Ihaka and Gentleman [1996] as a successor to S [Becker et al. 1988].

R's main data type is the primitive vector. Vectors are explicitly constructed by the constructor $c(\ldots)$, as in $c(1 L, 2 L, 3 L)$ which creates a vector of three integers. $R$ has a builtin notion of type that can be queried with the typeof function. Figure 1 lists all of the builtin types provided by the language; these are the possible return values of typeof. There is no intrinsic notion of subtyping in R, but in many contexts a logical will be coerced to integer, an integer will be coerced to double, and a double will be coerced to complex. Some odd conversions occur in corner cases, such as $1<" 2 "$ holds and $c(1,2)[1.6]$ returns the first element of the vector, as the double is converted to an integer. $\mathrm{R}$ does not distinguish between scalars and vectors (they are all vectors), so typeof $(5)==$ typeof $(c(5))==\operatorname{typeof}(c(5,5))==$ "double". All vectorized data types are monomorphic, except list which can hold values of any type, including list. For all monomorphic data types, attempting to store a differently-typed value will cause a conversion: Either the value is converted to the type of the vector, or vice versa.

All vectorized data types have a distinguished missing value denoted by NA (for "not available"). The type of NA is logical (typeof(NA)=="logical"), but NA inhabits every type: typeof $(c(1, N A)[2])==$ "double". $\mathrm{R}$ also has a NULL value. In data science, it is useful to have a notion of a "missing observation", since vectors are monomorphic there is a need for a missing value to inhabit each primitive type. In a sense, NA represents a missing data point, whereas NULL represents a missing data set.

Over time, programmers have found the need for a richer type structure. R supports this with attributes. One may think of attributes as an optional map from names to values attached to any built in type. Attributes are used to encode various type structures, and they are queried with attributes. Using attributes, programmers can extend the set of types by tagging data. For example, take the vector of

\begin{tabular}{|c|c|}
\hline Vectors: & \\
\hline logical & vector of boolean values \\
\hline integer & vector of 32 bit integer values \\
\hline double & vector of 64 bit floating points \\
\hline complex & vector of complex values \\
\hline character & vector of strings values \\
\hline raw & vector of bytes \\
\hline list & vector of values of any type \\
\hline \multicolumn{2}{|l|}{ Scalars: } \\
\hline NULL & singleton null value \\
\hline S4 & instance of a S4 class \\
\hline $\begin{array}{l}\text { closure } \\
\text { environment }\end{array}$ & $\begin{array}{l}\text { function with its environment } \\
\text { mapping from symbol to value }\end{array}$ \\
\hline \multicolumn{2}{|l|}{ Implementation: } \\
\hline $\begin{array}{l}\text { special, builtin, } \\
\text { language, char, .. } \\
\text { externalprt, }\end{array}$ & $\begin{array}{l}\text { symbol, pairlist, promise } \\
\text {., any, expression, } \\
\text { bytecode, weakref }\end{array}$ \\
\hline
\end{tabular}

Fig. 1. Builtin Types four values, $x<-c(1,2,3,4)$ and attach attribute dim, $\operatorname{attr}(x, " \operatorname{dim} ")<-c(2,2)$, to treat $x$ as a $2 x 2$ matrix. 
Another attribute that can be set is the class, which is one way to use R for object-oriented programming. The class attribute can be queried with class, and it can be bound to a list of class names: For instance, class $(x)<-c$ ("human", "friend") will set the class of $x$ to be human and friend. There are three object-orientation frameworks in R: S3, S4, and R5. The S3 object system support single dispatch on the class of the first argument of a function, whereas the $\mathrm{S} 4$ object system allows multiple dispatch. R5 allows for users to define objects in a more imperative style. Some of the most widely used data types leverage attributes, e.g., data frames and matrices. A data frame, for instance, is a list of vectors with a class and a column name attribute, and matrices are vectors with a dims attribute.

$\mathrm{R}$ functions have a number of quirks. Arguments can be assigned arbitrary expressions as default values; functions take variable numbers of parameters, and can be called positionally and nominally. Consider this example:

$$
f<- \text { function }(x, \ldots, y=i f(z==0) 1, z=0)\{x+y+\operatorname{if}(\operatorname{missing}(\ldots)) 0 \text { else } c(\ldots)\}
$$

This function has four formal parameters, $x$, dots, $y$ and $z$. Parameter $x$ can be bound positionally or passed by name. The dots, ..., is always positional. The remaining two parameters must be passed by name as they are preceded by dots; $y$ and $z$ have default values, in the case of $z$ this is a constant, but $y$ is bound to an expression that depends on $z$ 's value (if $z$ is not zero, $y$ defaults to NULL). The body of the function will add $x$ and $y$ to either 0 or the result of concatenating the dots into a primitive vector. The function missing tests if a parameter was explicitly passed. The following are some valid invocations of $f$ :

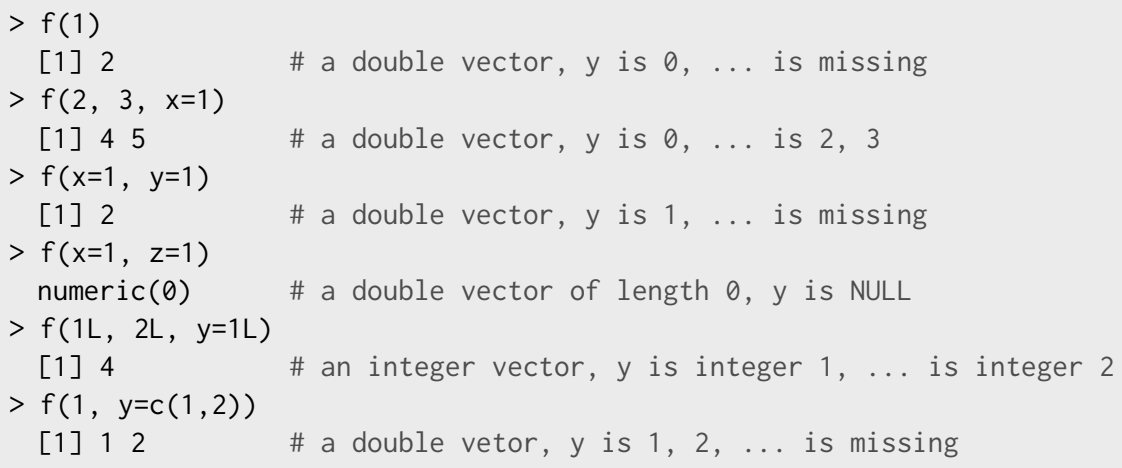

The above hints at polymorphism: $f$ may return a vector of integers or of doubles of length equal to the max length of its arguments.

\section{A TYPE LANGUAGE FOR R}

In this section, we set out to propose a candidate design for a type language to describe the signatures of $\mathrm{R}$ functions. The goal is not to propose a final design, but rather a starting point for an iterative process.

Given the peculiarities of the language there are a number of design choices that need to be reviewed and evaluated. It is not controversial to include types that cover the six kinds of primitive vectors, furthermore environments and the distinguished null types are commonly used and must be included. Environments are lists with reference semantics: mutating a value in an environment is performed in-place. They are used to store variables and to escape from the copy-on-write semantics of other data types. For simplicity, we omit some of the data types that closer to the implementation of the language. Fig. 2 presents our type language. 


\begin{tabular}{r|l}
$T::=$ & any \\
& null \\
& env \\
& $S$ \\
& $V$ \\
& $T \mid T$ \\
& $? T$ \\
& $\left\langle A_{1}, \ldots A_{n}\right\rangle \rightarrow T$ \\
& $\operatorname{list}\langle T\rangle$ \\
& $\operatorname{class}\left\langle I D_{1}, \ldots I D_{n}\right\rangle$
\end{tabular}

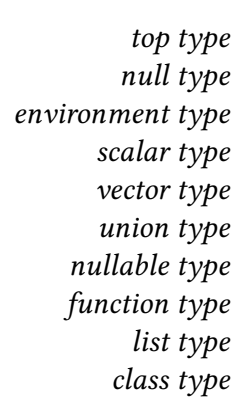

\begin{tabular}{|c|c|c|c|}
\hline$A$ & $::=$ & $T$ & arguments \\
\hline & 1 & .. & dots \\
\hline$V$ & $::=$ & $S[]$ & vector types \\
\hline & & ${ }^{\wedge} S[]$ & na vector types \\
\hline & $::=$ & int & integer \\
\hline & & chr & character \\
\hline & & $\mathrm{dbl}$ & double \\
\hline & & lgl & logical \\
\hline & & clx & complex \\
\hline & & raw & \\
\hline
\end{tabular}

Fig. 2. The R type language

Scalar. While R does not have scalar data types, there are cases where functions expect scalar values, for example a conditional takes a single logical and will complain if more values are passed. We considered tracking dimensions of data structures, but decided against it. Instead, the type language differentiates between vectors of lenght 1 and vectors of any dimension. The primitive types can be either vectors (e.g., int[] ) or scalars (e.g., int). A vector can happen to be of length 1 , which we handle with subtyping, shown later. Vectors are monomorphic, a vector of doubles contains only doubles.

Missing. Each primitive type has its specific NA. Many built-in functions, especially those implemented in $\mathrm{C}$ or Fortran, do not support NA values. It is thus advantageous to distinguish between vectors that can and cannot contain missing values. In our experience, functions that expect scalar values tend to not admit NAs, thus scalar types are treated as being NA-free. The type language allows one to write ${ }^{\wedge}$ int [ ] to specify that a vector of integers may have NAs and int [] to say that a vector must not have missing values. The type raw does not allow NAs, so ${ }^{\wedge}$ raw [] is NA-free. $R$ does not provide a built-in type testing mechanism, e.g., for the case of NA-free data types, it is necessary to scan vectors to find out if they have missing values.

Nullable. The null type is inhabited by a singleton NULL value often used as a sentinel. Unlike in some other lanugage, NULL is not the default value of uninitialized variables. $\mathrm{R}$ has different notion for that (which we do not cover here). To capture common uses of NULL, the type language has a nullable type, written ? T. Values of this type can be either values of type T or NULL.

Lists. Heterogeneous collections are implemented using lists. Lists and vectors are closely related: a vector converts to a list with as. list, and lists to vectors with unlist (coercions may ensue). The type language allows one to specify that a value is a list containing element of some type $T$, written list $\langle T\rangle$. R does not have built-in type tests for this purpose, to establish the type of a value requires traversal and checking individual elements.

Class. R has three objects systems, code-named S3, S4 and R5. All of them operate by adding a class attribute to values. The most widely used system by far is $S 3$, which supports single dispatch and multiple classes [Morandat et al. 2012]. The challenge from a type system point of view is that a value (such as an integer $5 \mathrm{~L}$ ) could be attributed with a class. Code that performs dispatch would use the class attribute while code that does not would view the value as an integer. The type language focuses on the attribute, and will hide the underlying type of the value. While this seems to match common usage, it does represent a loss of expressiveness. The type language also focuses on S3, and we leave the other object systems for future work. S3 has no notion of inheritance, each 
value has a list of classes. The type language thus allows to write class $\left\langle I D_{1}, \ldots, I D_{n}\right\rangle$ to denote values tagged with the class names $I D_{1}$ to $I D_{n}$.

Union. We support untagged unions of types written $T_{1}|\ldots| T_{n}$. The elements of unions are not disjoint, e.g., $1 \mathrm{~L}$ is both an int, a int [] and a ${ }^{\wedge}$ int [].

Function. Functions signatures the form $\left\langle A_{1}, \ldots, A_{n}\right\rangle \rightarrow T$ where each $A_{i}$ argument is either a type $T$ or dots (...), a variable length argument list. Moreover, a single function's signature can be the disjunction of a number of individual signatures.

Overall, the choice of type language follows the structure of values. The presence of NA-free data types and scalars are two choices that must be validated in practice. Note that nullable types are really just a special case of unions (i.e., ? $T$ is shorthand for $T \mid$ NULL).

\subsection{Subtyping}

$\mathrm{R}$ does not support the notion of subtyping between values, but we include a few simple rules in our framework to capture widespread coercion. The conversions between primitive types give us a starting point (e.g., an int is always accepted where a dbl is expected). Furthermore, the types introduced above induce some the rules of Figure 3: an NA-free vector is a subtype of its NA-ful equivalent, a value of type $T$ is a subtype of a nullable $T$, a list is subtype of another list if their elements are subtypes, and a scalar is a subtype of a vector of the same primitive type.

\begin{tabular}{|c|c|c|c|}
\hline$S[]$ & $<:$ & ${ }^{\wedge} S[]$ & \\
\hline$T$ & $<:$ & $? T$ & \\
\hline list $\langle T\rangle$ & $<:$ & list $\left\langle T^{\prime}\right\rangle$ & iff $T<: T$, \\
\hline$S$ & $<:$ & $S[]$ & \\
\hline lgl & $<:$ & int & \\
\hline int & $<:$ & $\mathrm{dbl}$ & \\
\hline $\mathrm{dbl}$ & $<:$ & clx & \\
\hline
\end{tabular}

Fig. 3. Subtyping

\subsection{Synthesizing Signatures}

Later in this paper, we will introduce a tool to synthesize function type signatures from running $\mathrm{R}$ code, wherein each invocation of a function will generate a trace representing the types of function arguments and returns. These traces will be combined into an overall arrow type for the function, and in order to keep these signatures compact we compact the traces into a single top-level arrow with unions at each argument position. Thus, the shape of function signatures will be:

$$
\left\langle T_{1,1}\left|T_{1, i}, \ldots, T_{n, 1}\right| T_{n, j}\right\rangle \rightarrow T_{1}|\ldots| T_{k}
$$

In other words, we take the union of the types occurring at individual argument positions rather than an union of function types. Furthermore, we apply some transformation on the types to keep the size of types in check. Figure 4 overviews the main simplification rules we adopted.

Assuming that type sequences can be reordered freely, we rewrite types to minimize their size by removing redundant types, types that are subsumed by subtyping, immutable lists, and replace null types with nullables. Higher-order functions are conservatively treated as any $\rightarrow$ any.

It is noteworthy that by performing this compaction we are losing precision, and the synthesized signatures will suggest combinations of argument types that were not observed. 


$$
\begin{aligned}
T \mid T & \Rightarrow T & \\
T \mid T^{\prime} & \Rightarrow T & \\
\text { list }\langle T\rangle \mid \operatorname{list}\left\langle T^{\prime}\right\rangle & \Rightarrow \text { list }\langle T\rangle & \\
\text { null }\left|S_{1}[]\right| \ldots S_{n}[] & \Rightarrow{ }^{\prime} S_{1}[] \mid \ldots S_{n}[] &
\end{aligned}
$$

Fig. 4. Simplification rules

\section{ANALYSIS AND INSTRUMENTATION PIPELINE}

For this paper, we have built tooling to $(a)$ automate the extraction of raw type signatures from execution traces, $(b)$ infer type signatures from a set of raw types, and $(c)$ validate the inferred

\begin{tabular}{|c|c|c|c|c|c|}
\hline Duration: & $1 \mathrm{~h}$ & $2 d^{1}$ & $10 \mathrm{~h}$ & $5 \mathrm{~h}$ & $5 \mathrm{~h}$ \\
\hline & $\begin{array}{l}\text { Create CRAN } \\
\text { mirror }\end{array}$ & $\begin{array}{c}\text { metadata } \\
\text { - code size } \\
\text { - code coverage }\end{array}$ & $\begin{array}{l}\text { Extract } \\
\text { raw type traces }\end{array}$ & $\begin{array}{l}\text { Infer } \\
\text { function type } \\
\text { sianatures }\end{array}$ & $\begin{array}{l}\text { Validate inferred } \\
\text { signatures }\end{array}$ \\
\hline Packages: & $15 \mathrm{~K}$ & 412 & 412 & 412 & running: 8,694 \\
\hline Code size: & $31.6 \mathrm{M}$ & $1.3 \mathrm{M}$ & running: $385 \mathrm{~K}$ & $\mathrm{~N} / \mathrm{A}$ & running: $19.6 \mathrm{M}$ \\
\hline Size: & package: $8 \mathrm{~GB}$ & packages: $1 \mathrm{~GB}$ & traces: $604 \mathrm{~GB}$ & types: 5MB & $\mathrm{N} / \mathrm{A}$ \\
\hline
\end{tabular}
signatures by the means of contracts. Figure 5 shows an overview of this pipeline. This section gives details of the main steps. The pipeline is run using GNU parallel [Tange et al. 2011] on Intel Xeon $6140,2.30 \mathrm{GHz}$ with 72 cores and $256 \mathrm{~GB}$ of RAM.

Fig. 5. The Analysis Pipeline; ${ }^{1}$ metadata has to be extracted for all CRAN packages.

\subsection{Types from Traces}

We implemented Typetracer, an automated tool for extracting types from execution traces of $\mathrm{R}$ programs. The goal of this tool is to output a tuple $\left\langle f, t_{1}, \ldots, t_{n}, t\right\rangle$ for each function call during the execution of a program, where $f$ is an identifier for a function, $t_{i}$ are type-level summaries of the arguments and $t$ is a summary of the return value.

While seemingly simple, the details and their proverbial devil are surprisingly tricky to get right at scale. Our implementation reuses R-dyntrace, an open source dynamic analysis framework for R [Goel and Vitek 2019] which consists of an instrumented R Virtual Machine based on GNU-R version 3.5.0. The framework exposes hooks in the interpreter to which user defined callbacks can be attached. These hooks include function entry and exit, method dispatch for the S3 and S4 object systems, the longjumps used by the interpreter to implement non-local exit, creation and forcing of promises, variable definition, value creation, mutation and garbage collection.

Types. The type information output by the tool includes the type tag of each value. Internal types are translated to names in the proposed type language. The next bit of information is the class, an optional list of names that may be absent, and, in some cases, is implicit (i.e. the interpreter blesses some values with the matrix and array classes even without attributes). Depending on a value's type, the tool collects further information: (a) for vectors, the presence of NA values, (b) for lists, element types by a recursive traversal, and (c) for promises, an approximation of the 
expression type. To obtain these types, we make use of R's C FFI and use low-level machinery to collect information from the R run-time. Types are completed during post-processing, and rely on the detailed information made available by these reflection mechanisms.

Promises. The fact that arguments are lazy (expressions are packed into promises and only evaluated on first access) complicates information gathering. For example, some promises may remain unevaluated, and it would be erroneous to force them as they may side-effect and change program behavior. To deal with unevaluated arguments, we make an initial guess for each argument at function entry and update the recorded type if the promise is forced.

Missing Arguments. Parameters which receive no values when the function is called are termed missing (not to be confused with NA). This occurs when a function is called with too few arguments and no default values are specified for those missing arguments. We record a missing type for such arguments. There are two obvious ways to deal with missing arguments: type them as any or type them as some unit type. We conservatively type them as any.

Non-local Returns. When a function exits with a longjump, there is no return value to speak of. To ensure call traces are valid when a longjump occurs, we intercept the unwinding process and record a special jumped return type for function returns that are skipped. As we cannot be sure of the intended return value, these jumped values become any types.

Dots. Arguments that are part of a dots parameter (denoted ...) are ignored. We do not attempt to give dots a type.

Implementation Details. We primarily rely on eight callbacks: closure_entry, closure_exit, builtin_entry, builtin_exit, special_entry, special_exit, promise_force_entry, and finally promise_force_exit. The function-related callbacks are used mainly for bookkeeping: the analysis is notified that a construct has been entered by pushing the call onto a stack. The calls themselves store a trace object that holds the type information. As R can perform single or multiple dispatch on function arguments depending on their class, the relevant information is kept by the _entry variants.

\subsection{Checking Signatures with Contracts}

One can validate a function's type signature by checking that it is respected in all programs that call the function. For this, we developed ContractR, an R package that decorates functions with assertions. We use it to insert type checking code around functions. For speed, ContractR's primary logic is implemented in C++. It has been tested with GNU R-3.5.0 and hardened with a battery of 400 test cases. An invocation of library (contractr) causes contracts to be injected. ContractR scans all packages in the workspace and inserts contracts in functions for which type signatures are available. Package load hooks are executed when new packages are loaded. ContractR automatically removes contracts from all functions and restores them to their original state when it is unloaded. The type signatures can be provided in an external file, thus avoiding the need to change the source code of checked packages. Type declarations can also be written in comments using Roxygen2 annotations, using the @type tag:

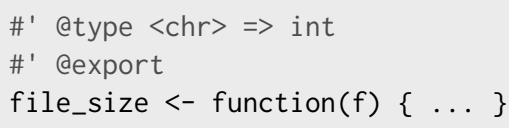

The injected contracts check arguments with a simple tag check when possible. Some properties require traversing data structures, such as the absence of NA. For union types, multiple checks may 
be needed, at worst one per member of the union. In order to retain the non-strict semantics of $\mathrm{R}$, the expression held in a promise is wrapped in a call to the type checker, and type checking is delayed until the promise is forced. This leads to corner cases such that the type checking of a function may happen after that function has returned. Return values require care as well. Functions return the last expression they evaluate, thus a callback is added to the exit hook. Another wrinkle is due to longjumps which causes active function calls on the stack to be discarded. When they are discarded, their exit hooks are called but they do not have a return value to type-check. ContractR deals with this problem by allocating a unique sentinel object which serves as the return value for calls that are discarded. The exit hook does not call the type-checker if it see the sentinel.

\section{PROJECT CORPUS}

Our aim is to gather data with which we can validate our type language design. To that end, we propose an experiment wherein we use Typetracer to synthesize types for some core corpus of packages, and validate those types by using ContractR and installing contracts on core corpus functions and running the client code of this corpus.

For this paper we have selected 412 packages consisting of $760.6 \mathrm{~K}$ lines of $\mathrm{R}$ code and $534.4 \mathrm{~K}$ lines of native code (C/Fortran). Figure 6 shows these packages: the size of the dots reflects the project's size in lines of code including both $\mathrm{R}$ and native code ${ }^{2}$, the $\mathrm{x}$-axis indicates the expression code coverage as a percentage and the y-axis gives the number of reverse dependencies in on log scale. Dotted lines indicate means. Packages with over 5K lines of code are annotated.

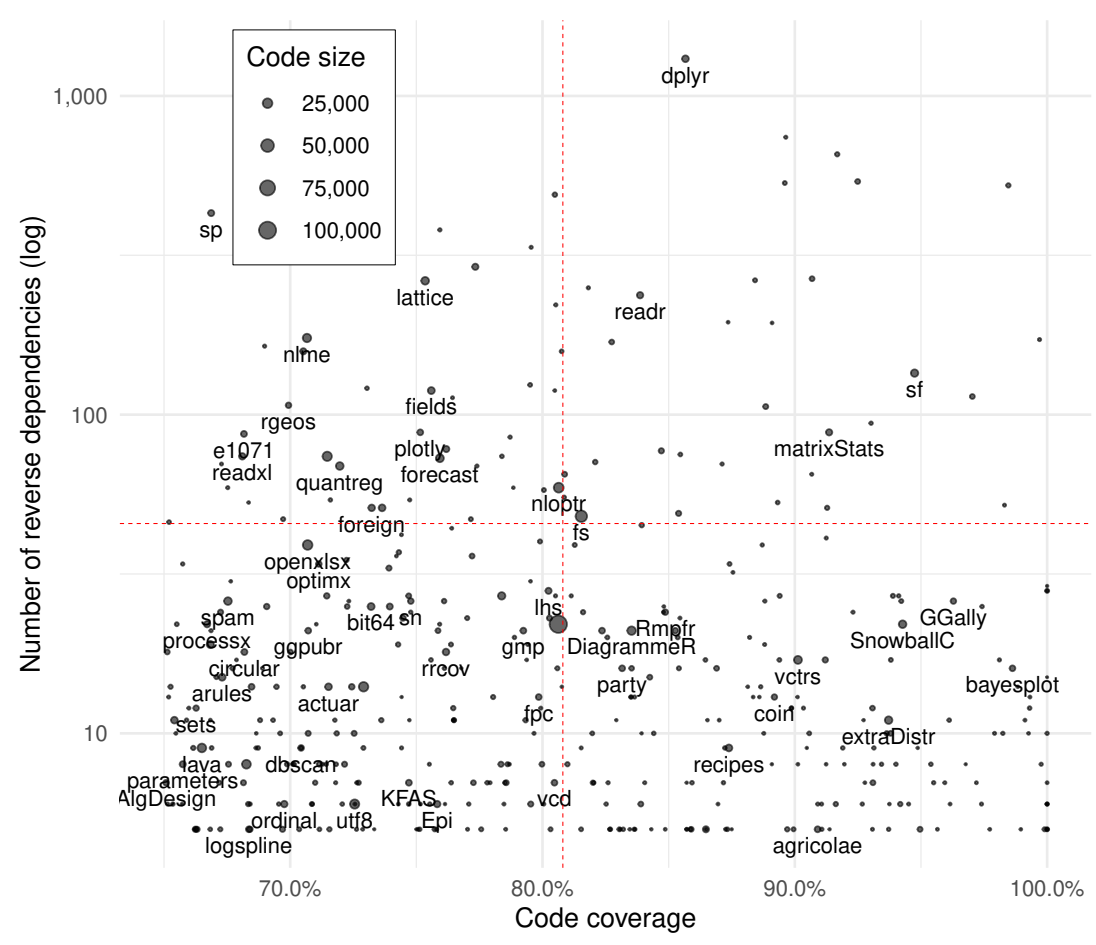

Fig. 6. Corpus

\footnotetext{
${ }^{2}$ Lines of source code reported excludes comments and blank lines, counted by $c l o c, c f$. https://github.com/AlDanial/cloc
} 
These packages come from the Comprehensive R Archive Network $\left(\mathrm{CRAN}^{3}\right)$, the largest repository of $\mathrm{R}$ code with over $15.4 \mathrm{~K}$ packages ${ }^{4}$ containing over $19.6 \mathrm{M}$ and $12.2 \mathrm{M}$ lines of $\mathrm{R}$ and native code respectively. Unlike other open code repositories such as GitHub, CRAN is a curated repository where each submitted package must abide by a number of well-formedness rules that are automatically checked to assess package quality. Notably, CRAN packages must have a set of runnable example, test, and vignette code which showcase package functionality. The code is run by CRAN, and only a successfully running package is admitted to the archive.

We have downloaded and installed all available CRAN packages. Out of the $15.4 \mathrm{~K}$ packages, we managed to install $13.5 \mathrm{~K}$. The main reason for this is that R-dyntrace (and, by extension, Typetracer) is based on GNU-R 3.5.0 and some of the packages are not compatible with this version. Some packages also require extra native dependencies which were not present on our servers.

We defined two criteria for including a package into the corpus: (1) the package must have runnable code that covers a significant part of the package source code from which type signatures could be inferred, and (2) the package must have some reverse dependencies that will allow us to evaluate the inferred types, using the runnable code from these dependencies. The concrete thresholds used were: at least $65 \%$ of expression coverage and at minimum 5 reverse dependencies. The code coverage was computed for each package using Covr ${ }^{5}$, the $\mathrm{R}$ code coverage $\mathrm{R}$ tool. The reverse package dependencies were extracted from the package metadata using built-in functions.

The 412 selected packages contain $385.8 \mathrm{~K}$ lines of runnable code in examples (98.9K), tests $(258 \mathrm{~K})$ and vignettes $(28.9 \mathrm{~K})$. Running this code results on average in $80.8 \%$ package code coverage (the average for all of CRAN is $65.6 \%$ ). Together, there is $18.8 \mathrm{~K}$ (on average 45.5 , median 12 ; CRAN average is 12.8 , median 2) reverse dependencies with $11.2 \mathrm{M}$ runnable lines of code resulting in $45.9 \%$ coverage (on average) of the corpus packages. Together there are $38.2 \mathrm{~K}$ defined $\mathrm{R}$ functions (17.4K are from the packages' public APIs). 11.8K are S3 functions, either S3 generics or S3 methods. Packages in the corpus define 81 S3 classes.

User Code. To represent end-user code in the corpus, we turned to Kaggle, an online platform for data science and machine learning. The website allows people to share data science competitions and data analysis problems together with data for which users try to find the best solution (something like a repository of hackathon or datathon code). The solutions, called kernels, are then posted on Kaggle either as plain scripts or as notebooks. One of the most popular competitions is about predicting passenger survival on Titanic ${ }^{6}$ with 2,890 kernels in R (over 1/4 of all available R kernels) which we used for our corpus.

Unlike CRAN, Kaggle is not a curated repository and therefore there are no guarantees about the quality of the code. After downloading all of the 2,890 kernels and extracting the R code from the various formats, ${ }^{7}$ we found that 1,079 were whole-file duplicates $(37.3 \%)$. From the resulting 1,811 kernels, 1,019 failed to execute. Next to various runtime exceptions, common problems were missing libraries (no longer available for R 3.5), parse errors, and misspelled package names. The final set contains 792 kernels with $33.7 \mathrm{~K}$ lines of $\mathrm{R}$ code. The Kaggle kernels are used for additional validation of the inferred types.

Type Usage. During execution, 3,147 different types were observed. Classes are the most common types, accounting for roughly $31 \%$ of types of arguments. The most common classes are matrices (12\%), data. frames ( $7.5 \%)$, formulas ( $2 \%$ ), factors ( $2 \%)$, and tibbles $(2 \%)$. Roughly $25 \%$ of classes are

\footnotetext{
${ }^{3}$ http://cran.r-project.org

${ }^{4}$ CRAN receives about 6 new package submissions a day [Ligges [n. d.]]

${ }^{5}$ https://github.com/r-lib/covr

${ }^{6}$ https://www.kaggle.com/c/titanic

${ }^{7}$ We use rmarkdown to convert from notebooks to R.
} 
part of R's base libraries, the others are user-defined. Scalars and vectors are the next most common kind, making up $41 \%$ of remaining types. with scalars making up $28 \%$ of types and vectors $12 \%$. Nulls and lists follow at $8 \%$ and $7 \%$ respectively, and the vararg type makes up $6 \%$ of arguments. This all totals up to over $90 \%$ of types. Table 1 reports on the 10 most frequent types occurring in the corpus. The first row of the table reads: the dbl type occurs in 12,298 (11.24\%) argument types, and accounts for over 12 million (20.3\%) of the types observed by Typetracer's dynamic analysis.

Table 1. Top types of arguments in $\mathrm{R}$

\begin{tabular}{lrrrr}
\hline Type & Args & \% of Args & Observations & \% of Obs. \\
\hline dbl & 12,298 & 11.24 & $12,152,787$ & 20.3 \\
lgl & 9,366 & 8.7 & $6,650,294$ & 11.1 \\
null & 8,799 & 8.0 & $2,187,611$ & 3.7 \\
chr & 8,727 & 8.0 & $2,564,207$ & 4.3 \\
dbl[] & 7,190 & 6.6 & $4,934,773$ & 8.2 \\
$\ldots$ & 6,611 & 6.0 & $6,075,874$ & 10.1 \\
any & 6,120 & 5.6 & 339,299 & 0.6 \\
chr[] & 4,325 & 4.0 & $1,060,466$ & 1.8 \\
class $\langle$ matrix & 4,152 & 3.8 & $2,805,718$ & 4.7 \\
class $\langle$ data.frame $\rangle$ & 2,608 & 2.4 & 352,655 & 0.6 \\
\hline
\end{tabular}

\section{EVALUATION}

We ran Typetracer on the test, example, and vignette code of the aforementioned corpus of 412 packages and successfully inferred types for 25,215 functions. Table 2 illustrates the process with ten representative signatures. Many of the features of our type language are represented here, and some signatures are telling of the function's behavior. For example, consider decrypt_envelope: the first three parameters of the function are byte arrays, and the fourth argument is an RSA key, used to decrypt some of the inputs, and the output of the function is another byte array. As another example, consider Traverse: according to the function documentation, it takes the root of a tree and traverses it in an order specified by the second argument. We see that reflected in the type, where the first argument has type class $\langle$ Node, R6 $\rangle$ and the second argument had type $\operatorname{chr}[]$, representing the traversal order.

Table 2. Select Type Signatures

\begin{tabular}{|c|c|}
\hline Function & Type Signature \\
\hline dplyr: :group_i & $\langle$ class $\langle$ data. frame $\rangle, \ldots\rangle \rightarrow \operatorname{int}[]$ \\
\hline ents: : all. cumulants & $\langle$ class $\langle$ matrix $\rangle \mid \mathrm{dbl}[]\rangle \rightarrow \operatorname{class}\langle$ matrix $\rangle \mid \mathrm{dbl}[]$ \\
\hline test: : dip & $\langle\mathrm{dbl}[], \mathrm{chr} \mid \mathrm{lgl}, \mathrm{lgl}, \mathrm{dbl}\rangle \rightarrow \operatorname{class}\langle\mathrm{dip}\rangle \mid \mathrm{dbl}$ \\
\hline stabledist: : cospi2 & $\langle\mathrm{dbl}[]\rangle \rightarrow \mathrm{dbl}[]$ \\
\hline matrixcalc: :matrix.power & $\langle$ class $\langle$ matrix $\rangle, \mathrm{dbl}\rangle \rightarrow$ class $\langle$ matrix $\rangle$ \\
\hline data.tree: :Traverse & $\langle$ class $\langle$ Node, R6 $\rangle, \operatorname{chr}[]$, any, any $\rangle \rightarrow$ list $\langle$ any $\rangle$ \\
\hline openssl: : decrypt_envelope & $\langle\operatorname{raw}[], \operatorname{raw}[], \operatorname{raw}[], \operatorname{class}\langle k e y, r s a\rangle$, any $\rangle \rightarrow \operatorname{raw}[]$ \\
\hline dbplyr: : set_win_current_group & $\langle ? \operatorname{chr}[]\rangle \rightarrow ? \operatorname{chr}[]$ \\
\hline openssl: : sha256 & $\langle\operatorname{raw}[], ? \operatorname{raw}[]\rangle \rightarrow \operatorname{raw}[]$ \\
\hline forecast: : initparam & $\langle ? \mathrm{dbl}$, any, any, any, chr, chr, lgl, dbl[], dbl[], any $\rangle \rightarrow \mathrm{dbl}[]$ \\
\hline
\end{tabular}


This section attempts to evaluate how well the proposed type language is able to describe the actual type signatures of functions. For this we focus on how often there is a single type for a particular argument; this is because union types and any are less accurate (and would likely require a more refined notion of subtyping or parametric polymorphism). Then, we evaluate how robust the inferred signatures are by checking that they remain valid for other inputs. Lastly, we try to see if the current proposal would be useful to programmers by allowing them to remove ad hoc checks and providing useful documentation.

\subsection{Expressiveness}

The first part of our evaluation attempts to shed light on how good a fit our proposed type language is with respect to common programming patterns occurring in widely used $\mathrm{R}$ libraries.

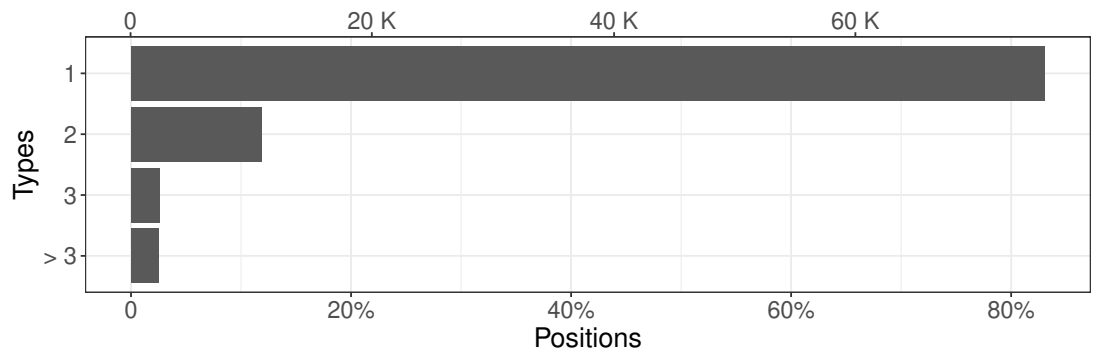

Fig. 7. Size of unions

First we look at the share of monomorphic arguments and function signatures. Monomorphic in this context means that the type is not relying on any or including a union. The import of monomorphism in this context is that it means our type language can accurately capture an argument's type or a function's signature. We get to that number in two steps. Fig. 7 shows the number of inferred argument types and their size (in terms of members of the union). The figure shows that most functions do not require a union at all (83.1\% of arguments do not have a union), and only $2.5 \%$ of positions have unions with more than three members.

Table 3. Singleton Type Categories

\begin{tabular}{lrrr}
\hline Types & Parameter \# & $\%$ & Cumulative \% \\
\hline scalar & 35064 & 33.33 & 33.33 \\
class & 24256 & 23.06 & 56.39 \\
vector & 13025 & 12.38 & 68.77 \\
$\ldots$ & 9142 & 8.69 & 77.46 \\
null & 7694 & 7.31 & 84.77 \\
any & 7614 & 7.24 & 92.01 \\
list & 3558 & 3.38 & 95.39 \\
^vector & 2923 & 2.78 & 98.17 \\
function & 1427 & 1.36 & 99.52 \\
environment & 500 & 0.48 & 100.00 \\
\hline
\end{tabular}

Table 3 provides a breakdown of types occurring in arguments without a union. Scalar, class and vector are the most common type categories. The shaded rows correspond to polymorphic 
types. When an argument's type is inferred to be null, we say that the argument is polymorphic due to a limitation in our analysis: In R, it is common for programmers to include default values for arguments, and in many cases this value happens to be NULL. Our type analysis will report a null type for these arguments if they are never passed a value during testing. We interpret these instances of null as polymorphic to capture that we cannot be sure of the actual type.

Removing the aforementioned instances of polymorphism gives us $68.9 \mathrm{~K}(60.4 \%)$ monomorphic positions in a corpus of $114 \mathrm{~K}$ parameters. With close to $60 \%$ of monomorphic argument or return values, it is fair to say that even a simple type language provides significant benefits.

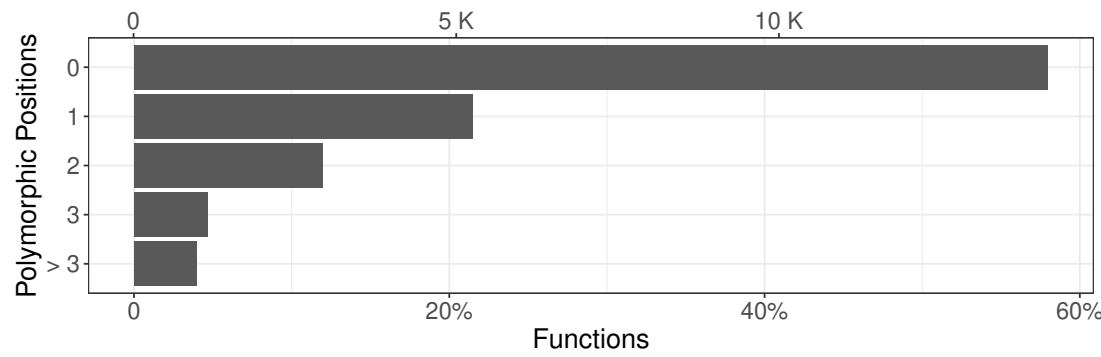

Fig. 8. Function Polymorphism

If we look at the numbers from the point of view of functions and count how many of their arguments are polymorphic, we observe that $58.0 \%(14.2 \mathrm{~K})$ functions are monomorphic. The remaining $42.0 \%$ (10.3 K) have at least one union or polymorphic parameter or return type. Figure 8 shows the distribution of functions against the number of polymorphic arguments. Finally, we count that 38 out of the 412 packages export only monomorphic functions.

\subsubsection{Discussion. A number of lessons can be drawn from the data we have gathered.}

NAs. Our data supports making the presence of NAs explicit. Only 2923 (or 2.78\%) of arguments are marked as possibly having NAs, thus the overwhelming majority of types appear to be NA-free. In practice, programmers check for them and sanitize them if they are present. Consider the binom package for computing confidence intervals and its binom. profile function. This attached code snippet highlights a data sanitization pattern: the programmer first binds the vectors into a matrix, then finds rows where both columns are not NA, extracts non-NA values and stores them into $x$ and $\mathrm{n}$ respectively.

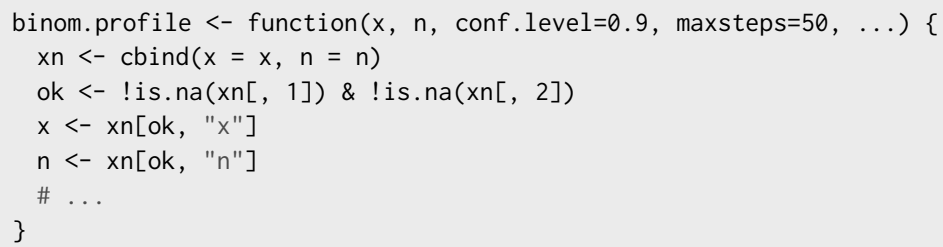

Scalars. The data also suggests that programmers often use scalars, and do dimensionality checks on their data. In our data 25,064 (or 33.33\%) of the arguments are scalar types. While not completely surprising, this is a rather large number. Consider the hankel.matrix function, it takes two arguments and checks that $\mathrm{n}$ is int, that $\mathrm{x}$ is a vector, and also, indirectly, that $\mathrm{n}$ is a a scalar (this comes from the fact that it is used in the guard of a conditional which fails if $n$ is not a scalar). 


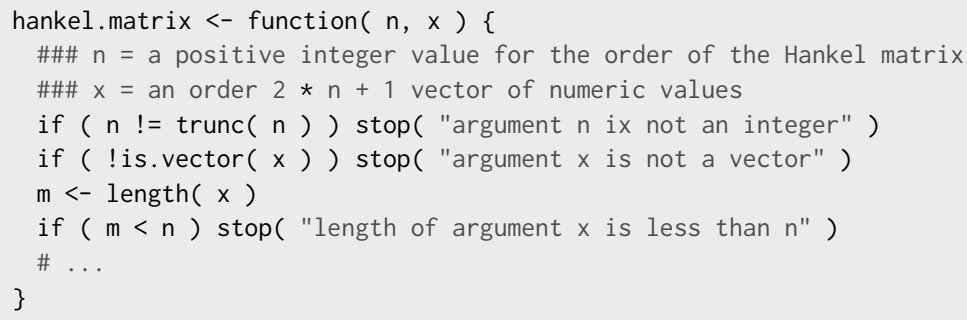

Nullables. The number of argument which may be NULL is 5057 (or 4.44\%). This is a relatively small number of occurrences, but it is worth expressing the potential for the presence of NULL as these would likely inhibit optimizations.

Higher-Order Functions. Typetracer assigns the type class〈function〉 to function values. The number of positions that receive a function (possibly as part of a union) is 1,705 , which is just $1.51 \%$ of all the positions for which we infer types. Given the small number of occurrences, it is not worth complicating the inferred types with a complete signature for these functions.

Structs. While experimenting with various design, we consider adding a struct type to capture lists with named elements that can be accessed with the $\$$ operators. We ended up discarding those types as they grew large and were often only representative of the example data being manipulated. Consider function $\mathrm{cv}$.model, its argument $\mathrm{x}$ is observed to be of class $\langle$ aov, $1 \mathrm{~m}\rangle$ or class $\langle 1 \mathrm{~m}\rangle$. Internally, linear models are represented as lists with named elements. The pollution is illustrated by the lines after the function definition, where the data(sweetpotato) expression loads a sample data set to test the function. The fields of sweetpotato will be recorded when cv.model is called.

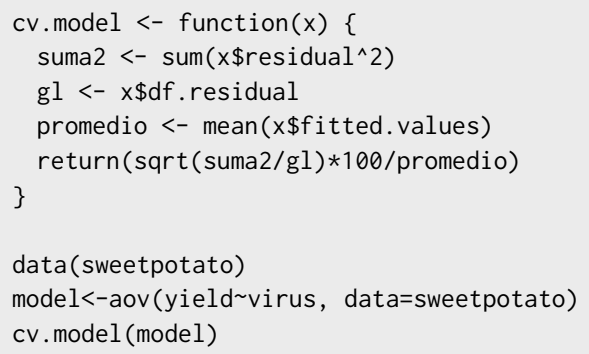

Objects. While we record classes, our analysis does not deal with method dispatch. $\mathrm{R}$ has multiple disparate object systems called S3, S4, and R5. The class attribute is used by these systems to dispatch methods. S3 does single dispatch, S4 does multiple dispatch, and R5 supports imperative objects. The mechanics of S4 dispatch are more complex than for S3, and users can define their own class hierarchies that we would need to incorporate in our type analysis and contract checking frameworks. We found limited use of S4 during our analysis. Coming up with a type system that accounts for all of these factors and consolidates multiple object-orientation frameworks in a single language design is an interesting problem in and of itself, one we leave for future work.

Matrices. Matrices are instances of the eponymous class, representing $10.71 \%$ of all classes occurring in types. They have a dims attribute indicating dimensions, and while not codified in the language semantics, many internal functions coerce vectors to matrices automatically. For example, the rowWeightedMeans function calculates the weighted means of rows. The programmer added a type check for $\mathrm{x}$. 


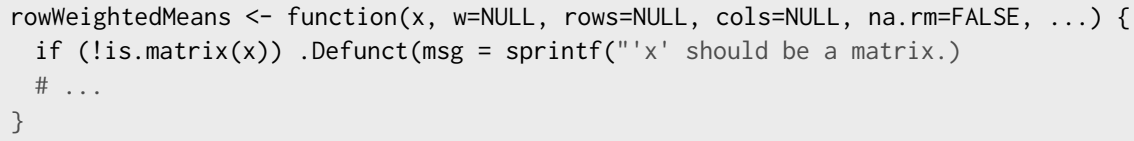

Data Frames. One of the most popular classes in $\mathrm{R}$ is the data. frame class, making up $8.15 \%$ of observed classes. Data frames and the derivative tibble and data.table types underpin much of the idiomatic usage of $\mathrm{R}$. One way to deal with data frames is through the struct type, with a named field for each column of the data frame, but as mentioned previously structs introduced undue noise. Further complicating data frames is that many functions built to operate on them operate in a name-agnostic way. For instance, the tidyverse package ecosystem allows programmers to pass column names to functions which operate on their data frames. In base R, typical data frame use is to use string column names to select rows from the frame (unless only a single column is of interest, wherein the $\$$ syntax is appropriate). In sum, data frames are a popular class of $\mathrm{R}$ values, and have spawned many derivative data types, such as tibbles and data tables. We include a class $\langle$ data.frame $\rangle$ type to cover most use-cases, and we leave a richer type for future work on a full fledged object-oriented type system.

\subsection{Robustness}

We now ask how robust are the inferred types? To measure this, we conducted another large-scale experiment: for each package in the corpus, using the inferred type signatures as contracts we ran all of the CRAN reverse dependencies for that package. In total we ran 8,694 unique packages and recorded 98,105,161 total assertions. Overall, we found that only $1.98 \%$ of contract assertions failed. The limit on number of arguments (we record only 20 ) accounted for $0.07 \%$ failed assertions. We found that $97.60 \%$ of parameter types and $87.70 \%$ of function types never failed. The number of immaculate function types increases to $89.70 \%$ if we discount S3 object method dispatch. Overall, these numbers are promising, and suggest that the type signatures are indeed robust.

We break down the failed assertions by type in Table 4 . Accounting for $36.36 \%$ of assertion failures are cases where $\mathrm{adbl}[]$ is passed where a class $\langle$ matrix $\rangle$ is expected. Considering these types, we might imagine them to be compatible, as a vector is just a one-dimensional matrix. However, not allowing this coercion was a deliberate design decision, as coercion of this kind is ad hoc at best, and unfortunately not a practice codified in the language. For example, if the vector has length $n$, should it be a $1 \times n$ or $n \times 1$ dimensional matrix?

In a similar vein, another popular failing assertion is checking if a dbl[] has type int[], another case of commonly performed coercion. We did not include these types of coercions in our type annotation framework as programmers cannot rely on them, and it is not always the case that the coercions are safe to perform.

The second row of the table is exemplary of a pattern where vectors are passed when scalars are expected. In these cases, the functions exhibiting these assertion failures were under-tested, and can operate just as well on vectors of values. As an example, this failure occurred in functions from the lubridate package which provides date/time functionality. Many functions, e.g., date_decimal and make_datetime turn doubles into class〈POSIXct, POSIXt) (which are dates in R), and they can easily operate on vectors of doubles, producing lists of dates.

Finally, we point out that assertion failures of, e.g., class $\langle$ data.frame $\rangle$ values being passed to class $\left\langle\right.$ data.frame, $\left.t b l, t b l \_d f\right\rangle$ arguments and class $\left\langle x m l \_n o d e\right\rangle$ values being passed to an argument expecting other XML-like classes are related to our simplified take on class types. Our 
Table 4. Top contract failures

\begin{tabular}{|c|c|c|c|c|}
\hline Passed & Arg Type & Occurrences & $\%$ Total & Cumul. \% \\
\hline $\mathrm{dbl}[]$ & class $\langle$ matrix $\rangle$ & 705,036 & 36.36 & 36.36 \\
\hline $\mathrm{dbl}[]$ & $\mathrm{dbl}$ & 189,800 & 9.79 & 46.15 \\
\hline chr & class $\langle$ bignum $\rangle \mid$ raw [] & 100,100 & 5.16 & 51.31 \\
\hline class $\langle$ simpleError, error, condition $\rangle$ & $\begin{array}{l}\text { class }\langle\text { data.frame }\rangle \mid \text { class }\langle\text { matrix }\rangle \mid \\
\text { class }\langle\text { randomForest }\rangle \mid \mathrm{dbl}[]\end{array}$ & 78,197 & 4.03 & 55.34 \\
\hline class $\langle$ data.frame $\rangle$ & class $\langle$ data.frame, tbl, tbl_df $\rangle$ & 58,809 & 3.03 & 58.37 \\
\hline class $\langle$ matrix $\rangle$ & class $\langle$ timeSeries $\rangle$ & 53,482 & 2.76 & 61.13 \\
\hline $\mathrm{dbl}[]$ & int [] & 33,350 & 1.72 & 62.85 \\
\hline $\mathrm{dbl}$ & class $\langle$ data.frame $\rangle$ & 32261 & 1.66 & 64.52 \\
\hline $\mathrm{dbl}[]$ & class $\langle$ data.frame $\rangle$ & 31361 & 1.62 & 66.13 \\
\hline class $\left\langle x m l \_n o d e\right\rangle$ & $\begin{array}{l}\text { class }\left\langle\text { xml_document, } x m l \_n o d e\right\rangle \mid \\
\text { class }\langle\text { xml_missing }\rangle \mid \text { class }\langle\text { xml_nodeset }\rangle\end{array}$ & 30,330 & 1.56 & 67.70 \\
\hline
\end{tabular}

type language does not encode user-defined subtyping and coercion, which could help address these mismatches.

In addition to the number of failed contract checks, we were interested in how many functions had a parameter where a contract check failed, and overall we found this to be the case in $12.29 \%$ of functions. To subdivide this number, we discounted functions that were performing S3 dispatch, as they exhibit user-defined polymorphism which we do not handle. Removing those functions, we see that the proportion of functions with failed contract checks falls to $10.30 \%$. These remaining functions were under-tested, as calls to these functions represent only $2.73 \%$ of recorded calls during Typetracer's run on the core corpus to infer types.

Turning our attention now to arguments, we found that only $2.40 \%$ of argument types failed. Table 4 showed the runtime occurrences, but that data alone does not tell the full story, as some failures may be overrepresented if, e.g., a failing contract assertion was in a loop. We were interested in knowing for each of the most common violations in Table 4, how many different arguments had that type, and how many of those exhibited the contract failure in question. Table 5 breaks down the failed assertions by type, folding away multiple identical failed contract assertions for the same parameter position. The first row of this table reads: a value of type $\mathrm{dbl}[\mathrm{]}$ was passed to 15 different function parameters expecting a class $\langle$ matrix $\rangle$, of which there are 1522 in total: 18 $(1.18 \%)$ of these class $\langle$ matrix $\rangle$-typed parameters were passed $\mathrm{dbl}[]$ values.

We see that even though the double vector and matrix issue was wildly prevalent in the dynamic contract evaluation numbers, the number of actual function argument types that were violated is very small. The story is similar with the double and integer coercion we mentioned earlier: it represents many dynamic contract failures, but very few of the int []-typed arguments have their contracts violated by $\mathrm{dbl}[]$-typed values. Row six is interesting: we see that rather often arguments expecting class $\langle$ timeSeries $\rangle$ data are passed class $\langle$ matrix $\rangle$ values. These are all from the timeSeries package, whose functions often accept matrices and vectors, converting them to time series in an ad hoc manner. Note that the code coverage of the timeseries tests, examples, and vignettes package code is only $58 \%$, which is one possible explanation for these contract failures: the types that Typetracer generates are only as good as the test code its run on.

Table 6 presents data on the most frequently violated contracts amongst the most frequently occurring argument types. We selected argument types which were in the 90th percentile of argument type occurrences, computed the most frequent type signature violations among them, and reported the most frequently violated contracts together with the type of the value that violated that contract. The first row of the table reads: 31 function arguments with int [] type are passed $\mathrm{dbl}[]$ values instead, and 624 arguments have int [] type, representing a failure rate of $4.97 \%$. 
Table 5. Results from Table 4, broken down by occurrences of the expected type as a parameter type

\begin{tabular}{|c|c|c|c|c|}
\hline \multirow[b]{2}{*}{ Passed } & \multirow[b]{2}{*}{ Arg Type } & \multicolumn{2}{|c|}{ \# Arg Types } & \multirow[b]{2}{*}{$\%$ Failure } \\
\hline & & Failed & Total & \\
\hline $\mathrm{dbl}[]$ & class $\langle$ matrix $\rangle$ & 18 & 1522 & 1.18 \\
\hline $\mathrm{dbl}[]$ & $\mathrm{dbl}$ & 66 & 5865 & 1.13 \\
\hline $\mathrm{chr}$ & class $\langle$ bignum $\rangle \mid \operatorname{raw}[]$ & 1 & 3 & 33.33 \\
\hline class $\langle$ simpleError, error, condition $\rangle$ & $\begin{array}{l}\text { class }\langle\text { data.frame }\rangle \mid \text { class }\langle\text { matrix }\rangle \mid \\
\text { class }\langle\text { randomForest }\rangle \mid \mathrm{dbl}[]\end{array}$ & 2 & 2 & 100 \\
\hline class $\langle$ data.frame $\rangle$ & class $\langle$ data.frame, tbl, tbl_df $\rangle$ & 23 & 196 & 11.73 \\
\hline class $\langle$ matrix $\rangle$ & class $\langle$ timeSeries $\rangle$ & 21 & 39 & 53.85 \\
\hline $\mathrm{dbl}[]$ & int [] & 31 & 624 & 4.97 \\
\hline $\mathrm{dbl}$ & class $\langle$ data.frame $\rangle$ & 2 & 1025 & 0.20 \\
\hline $\mathrm{dbl}[]$ & class $\langle$ data.frame $\rangle$ & 6 & 1025 & 0.59 \\
\hline class $\langle$ xml_node $\rangle$ & $\begin{array}{l}\text { class }\langle x m l \text { missing }\rangle \mid \text { class }\langle x m l \text { nodeset }\rangle \mid \\
\text { class }\langle x m l \text { document, } x m l \text { node }\rangle\end{array}$ & 1 & 1 & 100 \\
\hline
\end{tabular}

Table 6. Highest failure rate among popular argument types, for argument signatures whose frequency is in the 90 th percentile.

\begin{tabular}{llrrr}
\hline Passed & Arg Type & \# Args Failed & \# Args with Type & \% Failure \\
\hline dbl[] & int[] & 31 & 624 & 4.97 \\
dbl & int & 21 & 519 & 4.05 \\
chr[] & chr| null & 10 & 256 & 3.91 \\
^lgl[] & lgl[] & 8 & 219 & 3.65 \\
${ }^{\wedge}$ lgl & lgl[] & 5 & 219 & 2.28 \\
\hline
\end{tabular}

Table 7. Highest failure rate among popular argument types, for argument signatures whose frequency is in the 80 th percentile.

\begin{tabular}{|c|c|c|c|c|}
\hline \multirow[b]{2}{*}{ Passed } & \multirow[b]{2}{*}{ Arg Type } & \multicolumn{2}{|c|}{ \# Arg Types } & \multirow[b]{2}{*}{$\%$ Failure } \\
\hline & & Failed & Total & \\
\hline class $\langle$ matrix $\rangle$ & class $\langle$ timeSeries $\rangle$ & 21 & 39 & 35.90 \\
\hline $\mathrm{dbl}[]$ & class $\langle$ timeSeries $\rangle$ & 21 & 39 & 35.90 \\
\hline class $\langle$ data.frame $\rangle$ & class $\langle$ timeSeries $\rangle$ & 14 & 39 & 35.90 \\
\hline \multirow[t]{2}{*}{ class $\langle$ dtplyr_step_first, dtplyr_step $\rangle$} & class $\langle$ data.frame $\rangle$ & 10 & 45 & 22.22 \\
\hline & $\begin{array}{l}\text { class }\langle\text { data.frame, grouped_df, tbl, tbl_df }\rangle \mid \\
\text { class }\langle\text { data.frame, tbl, tbl_df }\rangle\end{array}$ & & & \\
\hline chr & raw & 6 & 31 & 19.35 \\
\hline
\end{tabular}

Had we failed to capture some key usage pattern of $\mathrm{R}$ with our type annotation framework, we would likely see it here. For example, consider Table 7, which was obtained identically to Table 6 except selecting arguments in the 80th percentile instead. The most frequent argument type violation pattern in that of class $\langle$ matrix $\rangle, \mathrm{dbl}[]$, and class $\langle$ data. frame $\rangle$ values passed to arguments expecting class $\langle$ timeSeries $\rangle$. This occurs in $35.90 \%$ of such arguments, and represents cases where tests did not adequately cover all valid function inputs. Separate from the issue of testing, we can capture this behavior with user-defined subtyping or coercion, as the data types which were passed are readily convertible to class〈timeSeries $\rangle$.

In sum, we believe that this evaluation shows that the type signatures we generate from traces are quite good. Only $1.98 \%$ of contract assertions failed at runtime, representing failures in as few as $2.40 \%$ of argument types. Even though $10.30 \%$ of functions had at least one argument type involved in a failing contract check, these functions are under-tested, representing only $2.73 \%$ of calls observed while inferring types. 
6.2.1 Other Observations. We drew a number of other observations from the contract assertion failure data.

First, we were curious about how many null-typed values flowed into non-nullable arguments, and we found that they accounted for $6.46 \%$ of contract assertion failures in 141 functions. We manually inspected some of the offending functions and observed three main patterns.

First, we found that many of these errors occurred in arguments that have a NULL default value. This would be the case when the programmer only tested the function by passing values to these null-by-default arguments, and clients of the package make use of the default. As an example, we observed a contract assertion failure when inner and labels were NULL in the following function:

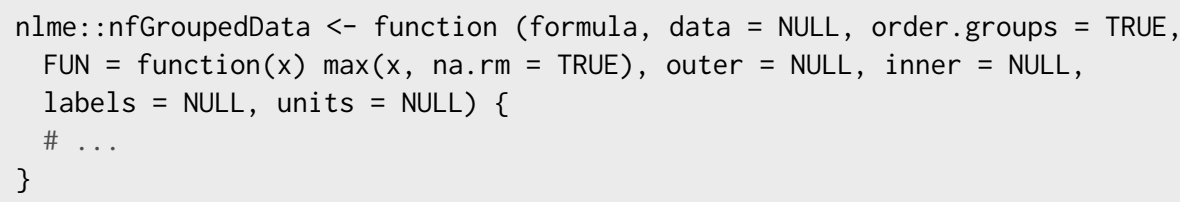

The other two patterns are for arguments with no default value, where either the call results in an error (perhaps explicitly handled by the programmer), or results in valid function behavior that was untested by the original package designer. Here, the first case can be explained by a lack of testing, and the second case is explained by programmers not fully understanding R's language semantics. For example, we observed this kind of error in the following function:

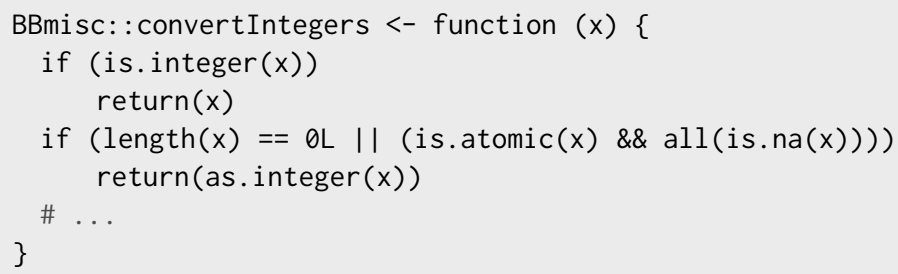

Here, if $x$ is NULL, the second branch of the conditional will be triggered (as in R, length (NULL) == 0 ), and the function will return as. integer (NULL), which curiously returns integer $(\theta)$, a zero-length vector of integers (one might expect it to return the integer NA value, or error).

Next, we analyzed how often vectors were passed to arguments expecting scalars. We found that $12.73 \%$ of dynamic contract assertion failures were of this type, and these errors were present in 114 functions. Besides an outright error, this kind of contract assertion failure might indicate that a function was not well-tested, in that it was only ever tested with unit-length vectors being passed to an argument which is intended to have a vector type. Further, these errors may reveal functions that were not designed with a vector-typed argument in mind, but can in fact handle vectors of values (in $\mathrm{R}$, most functions that can accept scalars can also accept vectors). As an example, consider the function BBmisc: : strrepeat, which takes a string and repeats it a specified number of times:

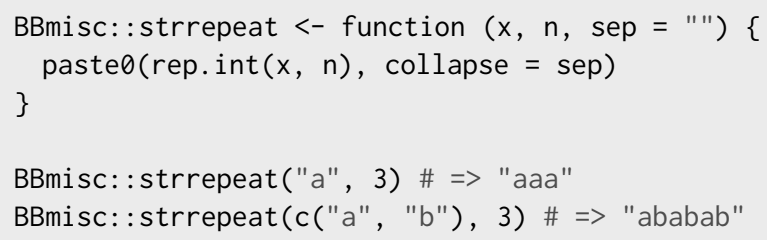

This function was only ever tested with unit-length vectors passed to $x$, even though technically it can handle longer vectors, as per the two sample calls above. This could be attributed to poor quality testing, or misunderstanding language semantics (e.g., misunderstanding the semantics of 
paste 0 and rep.int), but we found other instances of type errors where the functions really ought to have been tested with the offending type, for instance:

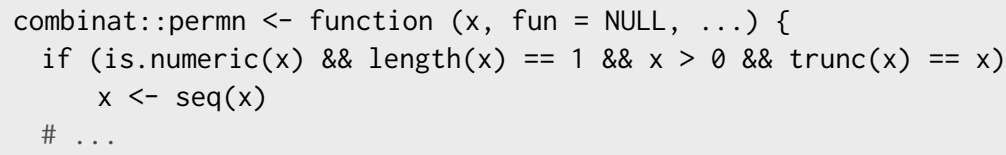

As per the documentation, this function is intended to take a vector, and generate all permutations of elements in that vector. If given a scalar integer $n$, it will generate all permutations for the list $[1,2, \ldots, n]$. Interestingly, the function was only tested by passing an integer, and not ever with a vector (even though, presumably, that is the main utility of the function).

Finally, we were curious to see what patterns of errors occurred in arguments expecting classes. Overall we found that $81.44 \%$ of assertion failures were on arguments which were expecting a class in some way (51.13\% of assertion failures were on monomorphic arguments expecting a class, the remainder on polymorphic arguments with at least one option being a class).

There are two broad divisions which account for most of the class-related contract assertion failures that are not outright errors. First, we observe a class of errors related to classes being passed to arguments expecting a different, yet convertible class. For instance, we observed class $\langle$ data.frame $\rangle$ values being passed to arguments expecting tibbles or data.tables (data frames have a straightforward conversion to these classes). Second, we observe a class of errors related more to coercion between simple data types and classes. As an example, consider the aforementioned assertion failures in the timeSeries package, and as a further example we found many instances of class $\langle$ matrix $\rangle$, class $\langle$ data.frame $\rangle$, and vectors being passed to arguments expecting class $\langle$ array $\rangle$, a generalization of matrices.

6.2.2 Kaggle. To further validate our inferred types, we repeated the experiment discussed in this section on end-user R code found on the Kaggle competition website.

By-and-large, this evaluation did not reveal any new insights, with the contract assertion failure patterns being repeated from the reverse dependencies. Overall, we observed that $2.14 \%$ of all contract assertions failed while running Kaggle code. If we remove assertion failures related to our simplifying assumption that function types will not have more than 20 arguments, that number drops to a mere $0.42 \%$. In all, $15.98 \%$ functions had at least one contract failure. There were $19,038,496$ assertions in total, on 970 functions.

Table 8. Top contract failures in Kaggle kernels

\begin{tabular}{|c|c|c|c|c|}
\hline Passed & Arg Type & Occurrences & $\%$ Total & Cumulative $\%$ \\
\hline class $\langle$ data.table, data.frame $\rangle$ & $\operatorname{class}\langle$ matrix $\rangle$ & 20002 & 28.15 & 28.15 \\
\hline class $\langle$ factor $\rangle$ & $\wedge \operatorname{chr}[]$ & 18344 & 25.81 & 53.96 \\
\hline class $\langle$ factor $\rangle$ & $\operatorname{chr}[]$ & $\begin{array}{r}10519 \\
7519\end{array}$ & 10.58 & 64.54 \\
\hline $\operatorname{chr}[]$ & $\begin{array}{l}\text { list }\langle\text { int }[]\rangle|\ldots| \\
\text { list }\langle\text { class }\langle\text { formula, quosure }\rangle\rangle\end{array}$ & 5385 & 7.58 & 72.12 \\
\hline class $\langle$ ixforeach, iter $\rangle$ & $\begin{array}{l}\text { class }\langle\text { dataframeiter, iter }\rangle|\ldots| \\
\text { class }\langle\text { iforeach, iter }\rangle\end{array}$ & 4139 & 5.82 & 77.94 \\
\hline
\end{tabular}

To mirror our analysis of contract assertions on the reverse dependencies of our corpus, we show in Table 8 the most frequently failing contract in Kaggle. While we don't see many overlapping entries per se, the assertions exhibit similar patterns. For instance, data tables (which have class (data.table, data.frame $\rangle$ ) are often passed to arguments expecting a class $\langle$ matrix $\rangle$. Data 
Table 9. Highest failure rate among popular argument types in Kaggle, for argument signatures whose frequency is in the 90 th percentile.

\begin{tabular}{llrrr}
\hline Passed & Arg Type & \# Args Failed & \# Args with Type & \% Failure \\
\hline chr[] & chr & 12 & 304 & 0.04 \\
class $\langle$ factor $\rangle$ & chr[] & 7 & 199 & 0.04 \\
dbl[] & $\operatorname{chr[]}$ & 6 & 199 & 0.03 \\
^chr[] & $\operatorname{chr[]}$ & 4 & 199 & 0.02 \\
int & chr & 5 & 304 & 0.02 \\
\hline
\end{tabular}

tables are essentially serve the same purpose as data frames and tibbles. As it happens, data tables can be coerced to matrices if their elements are unityped, and programmers will often interchange the two, as is the case here. One function producing many of these errors is the following:

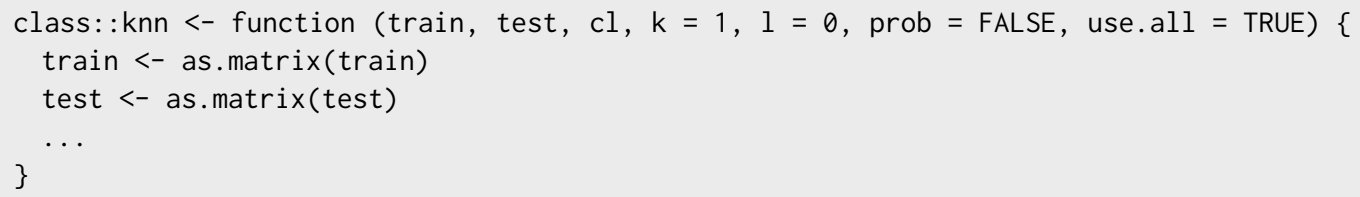

We see that class: : knn coerces its first two arguments to matrices. On the topic of coercion, rows two and three of Table are interesting as they depict factors being passed to arguments expecting character vectors. class $\langle$ factor $\rangle$ typed values are known as factors in $\mathrm{R}$, and they are stored as a vector of integer values corresponding to a set of character values, and their purpose is to allow for $\mathrm{R}$ to quickly deal with categorized data. Factors can be readily converted to characters when needed, as evidenced by these assertion failures.

Another interesting entry in Table 8 is the fifth row, where a class (ixforeach, iter is being passed to an argument expecting a long union of classes, each of the form class $\left\langle_{-}\right.$, iter $\rangle$. This is likely an instance where the user defined their own class, ixforeach, and wanted to use the iterators package (the user called iterator: : iter with a class $\langle$ ixforeach $\rangle$, and it gained the class iter on return). As mentioned, accounting for object-orientation in the type system is beyond the scope of this work, and such an inclusion would allow us to better type situations like this.

Table 9 mirrors Table 6 in showing contract violations on the most frequently occurring argument types. Here, our manual analysis has revealed similar failure patterns. In the case of vectors being passed to scalars, we find functions which can take vectors but were only tested with scalars (e.g., stringr: :str_to_upper which converts a vector of characters to upper case, and dplyr: : anti_join, which can join by a vector of column names but was only ever tested with a scalar). We also see possibly-NA character vectors being passed to NA-free character vectors. These assertion failures arise from a lack of testing: the offending functions are str_trim, str_sub, and str_replace_all from the stringr package. These functions are actually wrappers for other functions which have the correct argument type (^ $\mathrm{chr}[])$.

6.2.3 Discussion. Overall, the analysis discussed in this section has revealed two broad categories of contract assertion failures: those related to coercion, and those related to a class hierarchy. Our type system does not account for coercion as coercion in $\mathrm{R}$ is ad hoc at best, and it is implemented on a function-by-function basis, even in the core R packages. As for errors related to a class hierarchy, we aim to tackle this in future work, as designing a full fledged object-oriented type system for a language like $\mathrm{R}$ is outside of the scope of this work. 


\subsection{Usefulness of the Type Checking Framework}

There are a number of ways to check the types of function parameters in R. The default and most common way $^{8}$ is to use the stopifnot function from the R base package. It takes a number of $\mathrm{R}$ expressions which should all evaluate to TRUE, otherwise a runtime exception is thrown with a message quoting the failed expression. For example, the following code checks whether a given parameter $\mathrm{x}$ is a scalar string:

stopifnot(is. $\operatorname{character}(x), \operatorname{length}(x)==1 L$, ! is.na(x))

Beyond stopifnot, there are 4 packages in $\mathrm{CRAN}^{9}$ that focus on runtime assertions: assertive, ensurer, assertr, and assertthat. assertive and ensurer have not been updated since 2016 and 2015 respectively, and assertr is used by only 2 other packages and currently focuses on checking properties of data frames. Only assertthat is maintained and used (with 211 reverse dependencies). The advantage of assertthat over the $\mathrm{R}$ default is that it provides much better error messages.

One way to assess the usefulness of our type checking framework is to see how many existing user-defined type checks can be replaced by ContractR. To measure this, we extracted all calls to stopifnot and assert that assertions, and checked which among them could either be completely replaced by ContractR, or at least partially simplified by removing a portion of an assertion expression. Partial simplification is useful, as a common pattern in this ad hoc type checking is that the first part of the assertion checks the value's type, while the rest checks more detailed properties. In the example above, the whole expression could be replaced by a chr type check.

Out of the 412 packages, 153 use runtime assertions. Altogether, there are 1,995 assertions in 1,264 functions. Among these, ContractR can replace 1,005 (50.4\%) assertion calls across 114 packages and 688 functions. Furthermore, an additional 1,223 (61.3\%) asserts in 125 packages and 859 functions could have been simplified.

Checking the type of function parameters is not something that is seen often in the R code. In the whole of CRAN, there are only $32.3 \mathrm{~K}$ asserts in $15.9 \mathrm{~K}$ functions defined in $2.4 \mathrm{~K}$ packages. One may speculate that this is the case due to the verbosity and inconvenience of the existing assertion tools. Our system can infer type annotations for existing functions automatically. This can remove or partially remove over $61.3 \%$ of existing assertions.

\section{DISCUSSION}

Throughout this paper, we employed the following strategy to consolidate types: We collected all of the traces observed for a function, and combined them into a single function type, where the type for each argument position was made up of a union of all the observed types at that position. We then simplified these unions using the rules presented in Section 4. We call this an arrow of unions. We will elaborate on some of the design decisions that went into developing this method.

Arguably, the primary issue with the arrow of unions strategy is that of a loss of precision, as relationships between argument and return types can be obscured. For example, consider a function with two unique call traces, $\langle\mathrm{chr}\rangle \rightarrow \mathrm{chr}$ and $\langle\mathrm{dbl}\rangle \rightarrow \mathrm{dbl}$. The arrow of union strategy results in a combined type of $\langle\mathbf{c h r} \mid \mathbf{d b l}\rangle \rightarrow \mathrm{chr} \mid \mathrm{dbl}$ for the function, which hides the (potential) relationship between the argument and return type.

One solution to this is simply to convert each call trace into an arrow type, and generate a union of arrow types as the overall type for the function, dubbed the basic union of arrows strategy. This would lead to the type $\langle\mathrm{chr}\rangle \rightarrow \mathrm{chr} \mid\langle\mathrm{dbl}\rangle \rightarrow \mathrm{dbl}$ for the aforementioned function. Unfortunately, this leads to a significant blow-up in the size of types, and makes many types unreadable due to the

\footnotetext{
${ }^{8} 87.6 \%$ of all runtime checks in the whole of CRAN

${ }^{9}$ Packages are available on the CRAN website: https://cran.r-project.org/web/packages/
} 
myriad ways in which we combine the primitive types, as recall that, e.g., scalars and vectors are not the same type, and we see many types of the form $\langle\mathrm{chr}\rangle \rightarrow \mathrm{dbl}|\langle\mathrm{chr}[]\rangle \rightarrow \mathrm{dbl}|\left\langle{ }^{\hat{c}} \mathrm{chr}[]\right\rangle \rightarrow \mathrm{dbl}$ When comparing this consolidation strategy against the one employed throughout the paper, we find that the types using this strategy are much larger, with 14,057 (55.06\%) functions having at least one top-level union. We observed 93,229 independent arrow types using this strategy.

Another option is to employ a hybrid approach, wherein we perform the union of arrow types after grouping them together by return type (to further simplify, we also combine some primitive return types together, such as $\mathrm{dbl}$ and $\mathrm{dbl}[]$ ). While this has the advantage of reducing most of the blow-up of the previous approach, it suffers particularly when functions return classes, as our type system does not allow us to effectively consolidate class types. This strategy would reduce arrow types $\langle\mathrm{chr}\rangle \rightarrow \mathrm{dbl},\langle\mathrm{chr}[]\rangle \rightarrow \mathrm{dbl}$, and $\langle\hat{\mathrm{chr}}[]\rangle \rightarrow \mathrm{dbl}$ into the function type $\langle\hat{\mathrm{chr}}[]\rangle \rightarrow \mathrm{dbl}$ Comparing again to the strategy employed throughout the paper, we find 5,317 (20.82\%) functions to have a union of arrows, and we found 38,650 independent arrow types. We additionally find that $38,650(26.14 \%)$ of arrow types have at least one polymorphic argument.

These findings are summarized in Figure 9, which shows a breakdown of the number of toplevel alternatives in the function types obtained with both of these strategies: The term "top-level alternative" signifies an arrow type in the union of arrows, e.g., $\langle\mathbf{c h r}\rangle \rightarrow \mathrm{chr}$ and $\langle\mathbf{d b l}\rangle \rightarrow \mathrm{dbl}$ are top-level alternatives in the type $\langle\mathrm{chr}\rangle \rightarrow \mathrm{chr} \mid\langle\mathrm{dbl}\rangle \rightarrow \mathrm{dbl}$. We see that the hybrid approach greatly increases the number of functions with no union of arrows at the function level, where $92 \%$ of functions only have one or two top-level alternatives. Also, nearly $5 \%$ of function types obtained with the basic union of arrows strategy have over 9 top-level alternatives.

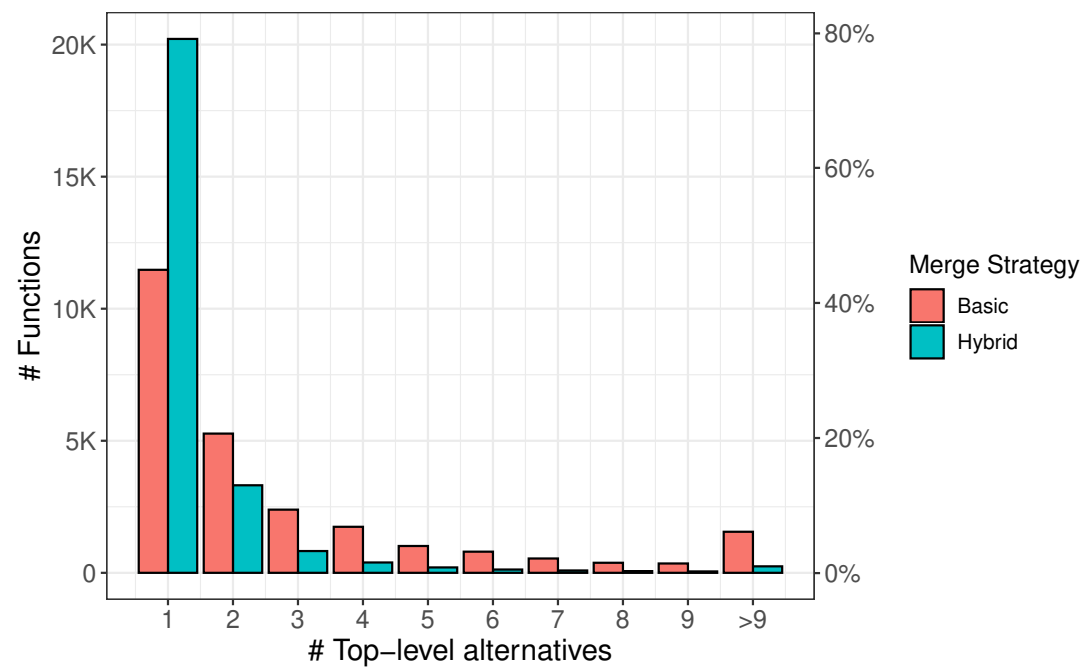

Fig. 9. Comparison of top-level function type counts across different merge strategies.

To connect this discussion with actual $\mathrm{R}$ types, we show what the types of the correlation function from the agricolae would be when using each of these strategies. agricolae: : correlation obtains the coefficients of correlation and $\mathrm{p}$-value between all variables of some input data table, using a method of the user's choosing. It returns the correlation matrix and the p-value (a double) together in a list. We refer the reader to Figure 10, where we see that the hybrid approach produces a much smaller type, with only two arrow types in the top-level union. Moreover, we see that no 
real precision is lost when further reducing the function type to a pure arrow of unions, as the two top-level alternatives in the type obtained with the hybrid approach are not very informative.

Table 10. Types of agricolae: : correlation with different type merge strategies.

\begin{tabular}{|c|c|}
\hline Merge Strategy & Function Type \\
\hline Union of Arrows & 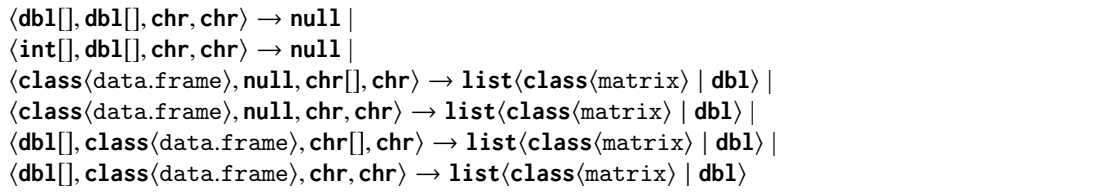 \\
\hline Hybrid & $\begin{array}{l}\langle\mathrm{dbl}[], \mathrm{dbl}[], \mathrm{chr}, \mathrm{chr}\rangle \rightarrow \mathrm{null} \mid \\
\langle\mathrm{class}\langle\text { data.frame }\rangle| \mathrm{dbl}[], ? \text { class }\langle\text { data.frame }\rangle, \mathrm{chr}[], \mathrm{chr}\rangle \rightarrow \operatorname{list}\langle\text { class }\langle\text { matrix }\rangle \mid \mathrm{dbl}\rangle\end{array}$ \\
\hline Arrow of Unions & $\langle$ class $\langle$ data.frame $\rangle|\mathrm{dbl}[], ? \mathrm{dbl}[]| \operatorname{class}\langle$ data.frame $\rangle, \operatorname{chr}[], \operatorname{chr}\rangle \rightarrow$ ? list $\langle$ class $\langle$ matrix $\rangle \mid \mathrm{dbl}\rangle$ \\
\hline
\end{tabular}

In contrast, there are real cases where a meaningful loss of precision occurs. Consider instead the rename function from the dplyr package, with types shown in Figure 11. This function takes a data frame and renames selected columns. We see that the types are the same with both the union of arrows and hybrid approach (with only three unique function signatures observed), and can glean from these types that dplyr: : rename produces a data frame of the same class as the input. This information is lost in the arrow-of-unions merge strategy. That said, once the type language is extend with proper user-defined type hierarchies, the arrow of unions type will be much smaller and more informative (as data frames, grouped tibbles, and tibbles are all related types).

Table 11. Types of dplyr: : rename with different type merge strategies.

\begin{tabular}{|c|c|}
\hline Merge Strategy & Function Type \\
\hline Union of Arrows & $\begin{array}{l}\langle\text { class }\langle\text { data.frame }\rangle, \ldots\rangle \rightarrow \text { class }\langle\text { data.frame }\rangle \mid \\
\langle\text { class }\langle\text { data.frame, grouped_df, tbl, tbl_df }\rangle, \ldots\rangle \rightarrow \text { class }\langle\text { data.frame, grouped_df, tbl, tbl_df }\rangle \mid \\
\langle\text { class }\langle\text { data.frame, tbl, tbl_df }\rangle, \ldots\rangle \rightarrow \text { class }\langle\text { data.frame, tbl, tbl_df }\rangle\end{array}$ \\
\hline Hybrid & $\begin{array}{l}\langle\text { class }\langle\text { data.frame }\rangle, \ldots\rangle \rightarrow \text { class }\langle\text { data.frame }\rangle \mid \\
\langle\text { class }\langle\text { data.frame, grouped_df, tbl, tbl_df }\rangle, \ldots\rangle \rightarrow \text { class }\langle\text { data.frame, grouped_df, tbl, tbl_df }\rangle \mid \\
\langle\text { class }\langle\text { data.frame, tbl, tbl_df }\rangle, \ldots\rangle \rightarrow \text { class }\langle\text { data.frame, tbl, tbl_df }\rangle\end{array}$ \\
\hline Arrow of Unions & $\begin{array}{l}\langle\text { class }\langle\text { data.frame }\rangle| \text { class }\langle\text { data.frame, tbl, tbl_df }\rangle \mid \text { class }\langle\text { data.frame,grouped_df, tbl, tbl_df }\rangle, \ldots\rangle \rightarrow \\
\text { class }\langle\text { data.frame }\rangle \mid \text { class }\langle\text { data.frame,tbl,tbl_df }\rangle \mid \text { class }\langle\text { data.frame, grouped_df,tbl, tbl_df }\rangle\end{array}$ \\
\hline
\end{tabular}

Finally, we want to briefly discuss types for base package functions (in R, functions like +, -, and vector access are part of the base package). These functions coerce mismatched arguments in an ad hoc manner, with no real defined semantics. Further, functions like + often act on values based on their type (according to typeof), and ignore the class, unless a package extended + to dispatch on the class. This leads to incredibly large inferred signatures for these functions. Even after counting out traces related to S3 and S4 dispatch, the type of + using the hybrid approach has 29 top-level alternatives, 22 of which have a class-typed argument. We don't believe this type to be particularly useful to a programmer, but a compiler might find it quite useful, as it can determine the type of the return value of a function given the types of its arguments.

\section{CONCLUSION}

Retrofitting a type system for the interactive and exploratory programming style of $\mathrm{R}$ is hard: The language is poorly specified and builds upon an eclectic mix of features such as laziness, 
reflection, dynamic evaluation and ad-hoc object systems. Our intent is to eventually propose a type system for inclusion in the language, but we are aware that for any changes to be accepted by the community, they must have clear benefits without endangering backwards compatibility. As a step towards this, we focus on a simpler problem: instead of an entire type system, we limited the scope of our investigation to ascribing types to function signatures. To this end, we designed a simple type language which found a compromise between simplicity and usefulness by focusing on the most widely used features of R. We presented Typetracer, a tool for inferring types for function signatures from runnable code, and ContractR, an easy-to-use package for $\mathrm{R}$ which allows users to specify function type signatures and have function arguments checked for compliance at runtime.

We evaluated our design by running Typetracer on a corpus of 412 of the most widely used $\mathrm{R}$ packages on CRAN, inferring signatures for exported functions, and testing those inferred signatures on the 8,694 reverse dependencies of the corpus. Overall, we found that our simple design fits quite well with the existing language: Nearly $80 \%$ of functions are either monomorphic or have only one single polymorphic argument. When we tested the types inferred by Typetracer during our evaluation, we found that only $1.98 \%$ of contract assertions failed. Furthermore, we found that our type language and contract checking framework would be useful to programmers, eliminate or otherwise simplify $61.3 \%$ of existing type checks and assertions in user code. In sum, we believe that our simple type language design is a solid foundation for the eventual type system for R.

\section{ACKNOWLEDGMENTS}

This work has received funding from the Office of Naval Research (ONR) award 503353, the National Science Foundation awards 1759736, 1544542, 1925644, and 1910850, the Czech Ministry of Education, Youth and Sports from the Czech Operational Programme Research, Development, and Education, under grant agreement No. CZ.02.1.01/0.0/0.0/15_003/0000421, and the European Research Council (ERC) under the European Union's Horizon 2020 research and innovation programme, under grant agreement No. 695412. This work was partially funded by NSERC.

\section{REFERENCES}

Miltiadis Allamanis, Earl T. Barr, Soline Ducousso, and Zheng Gao. 2020. Typilus: neural type hints. In Conference on Programming Language Design and Implementation (PLDI). https://doi.org/10.1145/3385412.3385997

Jong-hoon (David) An, Avik Chaudhuri, Jeffrey S. Foster, and Michael Hicks. 2011. Dynamic Inference of Static Types for Ruby. In Proceedings of the 38th Annual ACM SIGPLAN-SIGACT Symposium on Principles of Programming Languages. https://doi.org/10.1145/1926385.1926437

Esben Andreasen, Colin S. Gordon, Satish Chandra, Manu Sridharan, Frank Tip, and Koushik Sen. 2016. Trace Typing: An Approach for Evaluating Retrofitted Type Systems. In European Conference on Object-Oriented Programming (ECOOP). https://doi.org/10.4230/LIPIcs.ECOOP.2016.1

Richard A. Becker, John M. Chambers, and Allan R. Wilks. 1988. The New S Language. Chapman \& Hall, London.

Jeff Bezanson, Jiahao Chen, Ben Chung, Stefan Karpinski, Viral B. Shah, Jan Vitek, and Lionel Zoubritzky. 2018. Julia: Dynamism and Performance Reconciled by Design. Proc. ACM Program. Lang. 2, OOPSLA (2018). https://doi.org/10. $1145 / 3276490$

Gavin Bierman, Martin Abadi, and Mads Torgersen. 2014. Understanding TypeScript. In European Conference on ObjectOriented Programming (ECOOP).

Gavin M. Bierman, Erik Meijer, and Mads Torgersen. 2010. Adding Dynamic Types to $C^{\#}$. In European Conference on Object-Oriented Programming (ECOOP). https://doi.org/10.1007/978-3-642-14107-2_5

Michael Furr, Jong-hoon (David) An, and Jeffrey S. Foster. 2009. Profile-guided static typing for dynamic scripting languages. In OOPSLA. https://doi.org/10.1145/1640089.1640110

Aviral Goel and Jan Vitek. 2019. On the Design, Implementation, and Use of Laziness in R. Proc. ACM Program. Lang. 3 , OOPSLA (2019). https://doi.org/10.1145/3360579

Ross Ihaka and Robert Gentleman. 1996. R: A Language for Data Analysis and Graphics. Journal of Computational and Graphical Statistics 5, 3 (1996), 299-314. http://www.amstat.org/publications/jcgs/ 
Uwe Ligges. [n. d.]. 20 Years of CRAN (Video on Channel9. In Keynote at UseR!

André Murbach Maidl, Fabio Mascarenhas, and Roberto Ierusalimschy. 2014. Typed Lua: An Optional Type System for Lua In Workshop on Dynamic Languages and Applications (DyLa). https://doi.org/10.1145/2617548.2617553

Floréal Morandat, Brandon Hill, Leo Osvald, and Jan Vitek. 2012. Evaluating the Design of the R Language: Objects and Functions for Data Analysis. In European Conference on Object-Oriented Programming (ECOOP). https://doi.org/10.1007/ 978-3-642-31057-7_6

Python Team. 2020. Type Hints for Python. https://docs.python.org/3/library/typing.html.

Ole Tange et al. 2011. Gnu parallel-the command-line power tool. The USENIX Magazine 36, 1 (2011).

Sam Tobin-Hochstadt and Matthias Felleisen. 2008. The design and implementation of typed Scheme. In Symposium on Principles of Programming Languages (POPL).

Julien Verlaguet. 2013. Hack for HipHop. CUFP, 2013, http://tinyurl.com/lk8fy9q.

Tobias Wrigstad, Francesco Zappa Nardelli, Sylvain Lebresne, Johan Östlund, and Jan Vitek. 2010. Integrating typed and untyped code in a scripting language. In Symposium on Principles of Programming Languages (POPL). https: //doi.org/10.1145/1706299.1706343 